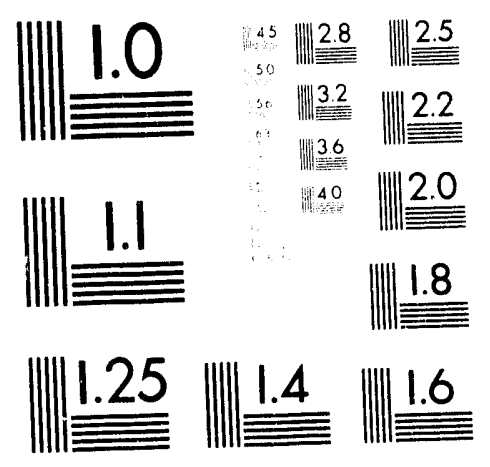



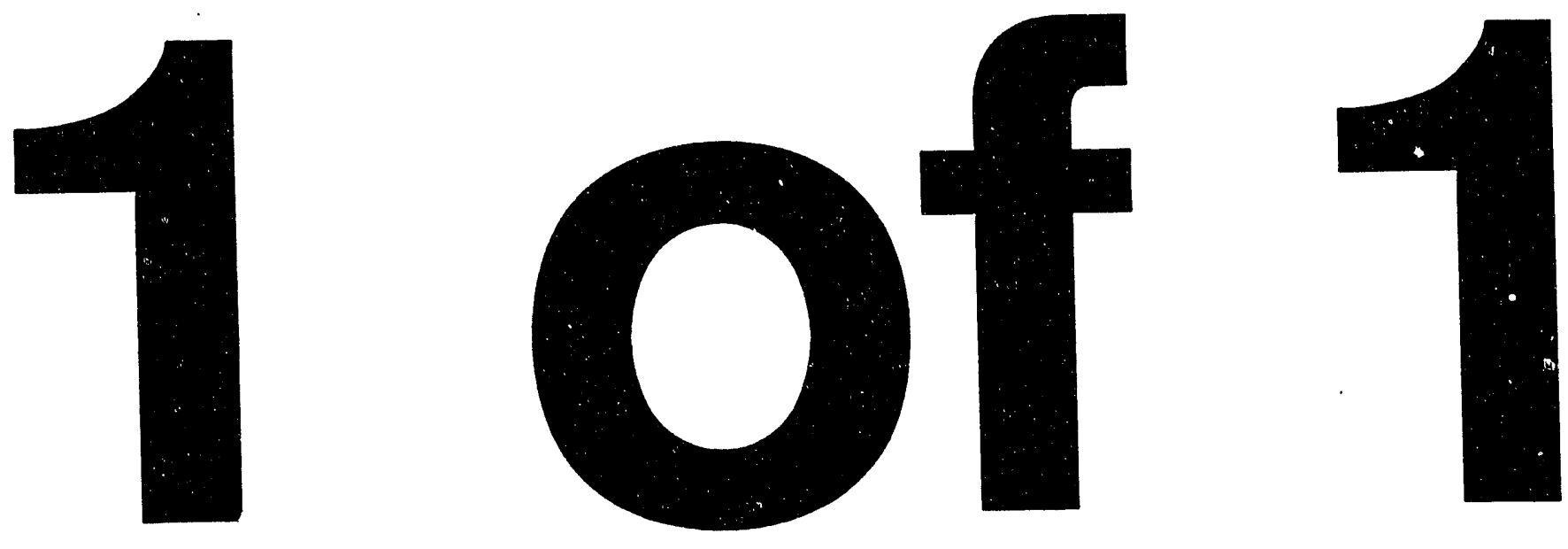


\title{
Radio Galaxies and their Environment
}

\author{
Wil van Breugel
}

This paper was prepared for submittal to the Jets in Extragalactic Radio Sources

Rinberg Castle, Germany

September 22-28, 1991

February 24, 1993

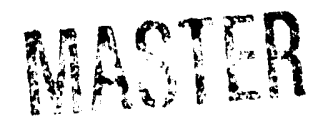

242

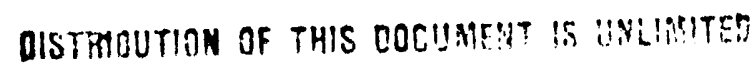




\title{
Radio Galaxies and their Environment
}

\author{
Wil van Breugel \\ Institute of Geophysics and Planetary Physics \\ L-413, Lawrence Livermore National Laboratory \\ P.O. Box 808 \\ Livermore, CA 94551-9900, LiSA
}

"What you see is what you get"

Wild Bill Hickock

\section{Introduction}

The relationships between radio galaxies and their environment are varied, complex, and evolve with cosmic epoch. Basic questions are what role the environment plays in triggering and fuelling (raciri) galaxy activity, what the effects of this activity are on its environment, and how radio galaxies and environment evolve. Clearly, this could be the topic of a workshop all in itself and the scope of this review will necessarily be limited. A review of the connections between environment and galaxy activity in general has been given by Heckman (1990). .

First, I will briefly summarize the relationships between parent galaxy and cluster environments, and radio galaxies. A more detailed discussion of various aspects of this will be given elsewhere by F. Owen, J.O. Burns and R. Perley. I will then discuss the current status of investigations of extended emission-line regions in radio galaxies, again referring elsewhere in this volume for more detailed discussions of some particular aspects (kinematics and ionization mechanisms by $\mathrm{K}$. Meisenheimer; polarization and spectral index lobe asymmetries by G. Pooley). I will conclude with a brief discussion of the current status of observations of high redshift radio galaxies.

\section{Parent Galaxies, Neighbourhoods and Cluster Environments}

Optical observations have shown that a large fraction $(30 \%$ to $50 \%)$ of nearby $(z<0.5)$, powerful $\left(P_{1100, \text { rest }}>10^{33} \mathrm{ergs} \mathrm{Hz}^{-1} \mathrm{~s}^{-1}\right)^{22}$ FR-II type radio galaxies exhibit peculiar optical features (tails, fans; Heckman et al. 19S6; Hutchings 1987) and unusually blue, extra-nuclear colors (Smith and Heckman 19S9). While their $r^{-1 / 4}$ surface brightness profiles still classify them as ellipticals, the morphological distortions strongly suggest

${ }^{22} H_{0}=50, q_{0}=0.0$ 
that they are interacting or merging with nearby gas rich companions (see Toomre and Toome 1072). Support for this also comes from stellan dyanics studies which show that poverful radio galaxies which are morphologically peculiar exhibit more stellar rotation than other radio galaxies and normal ellipticals. presumably because of recent merging with a disk galaxy (Smith et al. 1990).

On the other hand. Low power $\left(P_{1+00 \text { rest }}<10^{33}\right.$ ergs $\left.\mathrm{Hz}^{-1} s^{-1}\right)$. FR I type radio galaxies appear associated with relatively normal elliptical galaxies, albeit generally of the D or cD type (e.g. Owen and Laing 1989). In addition to this morphological difference between the parent galaxies of low and high power radio sources. investigations reported at this workshop by $F$. Owen further suggest that the radio FR I / FR II rype break may be a function of optical luminosity as well.

Several studies have been made of the galaxian/cluster envin onments of radio galaxies (e.g. Hecliman, Carthy and Bothun 1985: Yates, Miller and Peacock 1959: Smith and Hechman 1990; Hill and Lilly 1991). These stuclies have shown that at low redshifts. powerful FRII type radiogalaxies inhabit relatively sparsely populated regions with average galaxy densities a factor 2-3 lower than those of low power FR I type radio galaxies. At larger redshifts $(z \gtrsim 0.5)$. the cluster environments of FR II type radio galaxies appear to be richer, while no change in environment has been found for FR I types (Hill and Lilly 1991). This suggests that FRII actirity may somehow be related to the evolution of its cluster environment (intergalactic medium. galaxy richness, or both). Incidentally, the cluster environments of powerful radio galaxies and quasars are rery similar (Smith and Heckman 1990; Hill and Lilly 1991), suggesting that these types of objects are also closely related.

While much about the detailed interpretation of these results remains unclear, the difference in parent galaxy types, envirommerts, and stellar kinematics suggests that the two classes of radio sources do not simply evolve into one another, at least not on typical FRII timescales which may be at most a few times $10^{\top}$ years (Leahy 1991). The evidence strongly suggests that the interaction and merging of elliptical galaxies with gas rich companions play's an important role in triggering or fuelling powerful, FR II type radio source activity. The resulting decrease in galaxy density surrouncling the radio galaxies would be consistent with the observed decrease in cluster richness with redshift. The importance of galaxy interactions in connection with galaxy actirity and central star formation is being recognized in a variety of galaxy types. including star burst galaxies, Seyferts and quasars, as well as radio galaxies (see Heckman 1990). The processes of galaxy activity and star formation may occur simultaneously; or may even be causaly connected. When dense gas rapidly accumulates in the central regions of the interacting galaxies (Norman and Scorille 1988: Hernquist 1059: Shlosman. Begelman and Frank 1990).

The lower radio luminosities of the less disturbed parent galaxies of FR I type radio souces suggest a different origin and erolution of their activity. In these cases the "gassupply" to their galaxy nuclei may be due to infall from their halos or clusters (cooling fows). and is presumably more seclate. continuous and of a lower level than is provided my the rapid merging processes thought to occur in FR IIs. 


\section{Interaction of Radio Galaxies with Their Environment}

\subsection{Extended Emission-Line Regions}

Extended emission-line regions (EELRs) in radio and other active galaxies are being
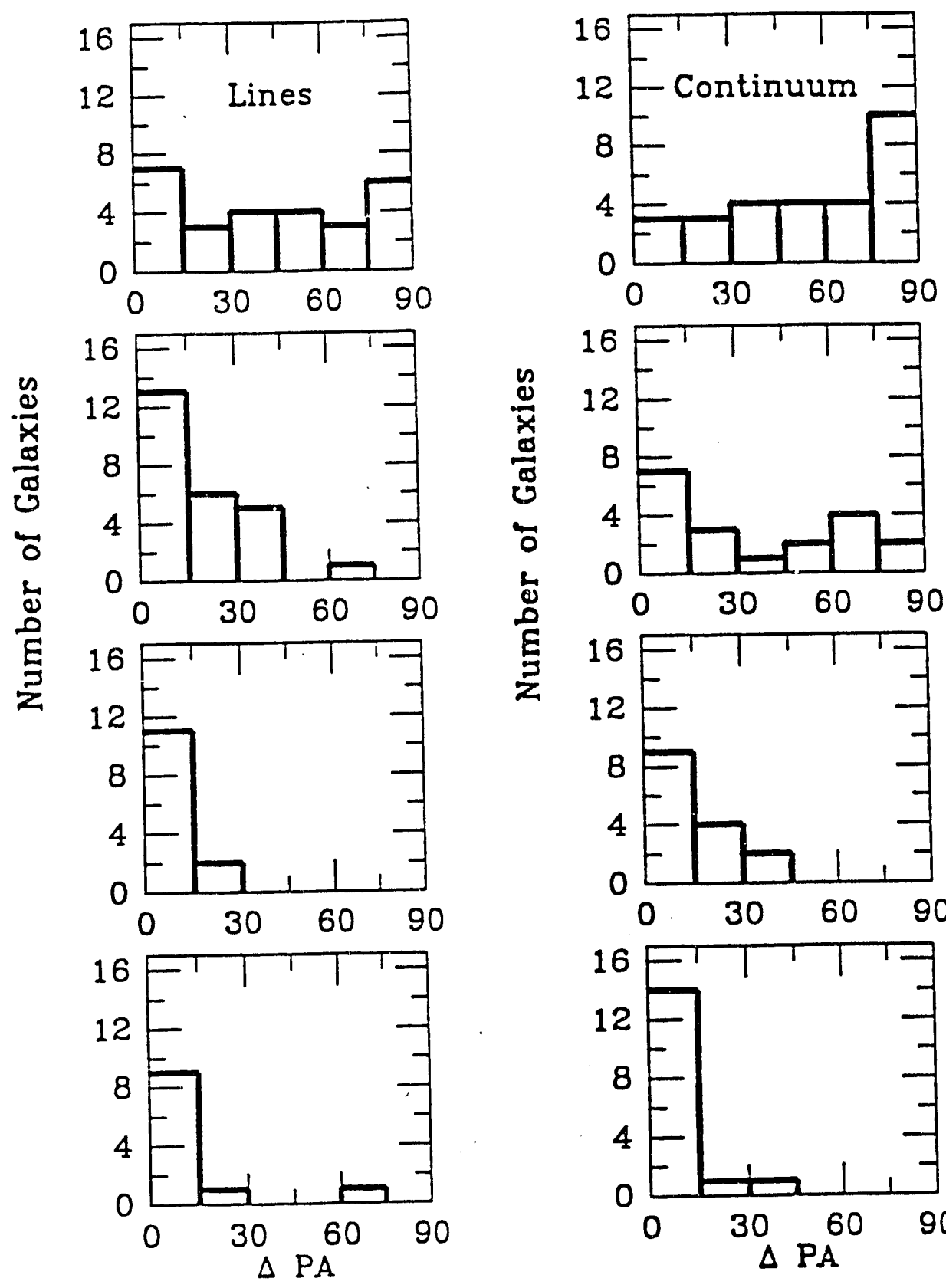

$0<\mathrm{Z}<0.1$
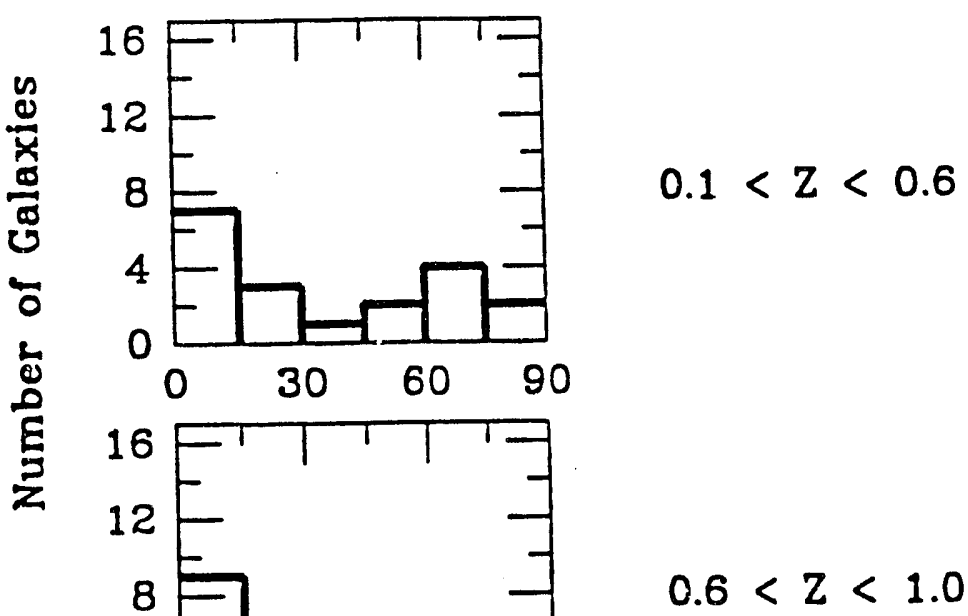

Fig. 1. These histograms show the inceasingly better alignment of EELR (left panels) and optical continuum emission (right panels) as function of redshift/radio power in the $3 \mathrm{CR}$ sample studied by McCarthy (1989).

studied by many groups. Important new developments since the last Ringberg workshop in 1988 are the realization that emission-line "cones" may be ubiquitous in active galax- 
ies (Seyferts and radio galaxies), and the extremely good correlation between EELR and radio lobe asrmmetries. I briefiy summarize the current status and i efer for a more detailed discussion of many relevant aspects to my previous review (van Breugel 1988), and to the proceedings of the ESO workshop on "Extranuclear Activity in Galaxies" (1989).

\subsubsection{Occurrence}

Narrow-band surveys of representative samples of nearby and distant radio galaxies show that a large fraction $(\sim 70 \%$ ) hare extended optical emission line regions (e.g. Baum and Hechman 1989a,b; .IcCarthy, Spinrad and ran Breugel 1992).

\subsubsection{Alignments}

In relatively powerful radio galaxies the EELRs are usually within $\sim 20^{\circ}$ of the radio axes, in most cases between the galaxy nucleus and radio hotspots (Baum, Heckman and van Breugel 1990; McCarthy; Spinrad and van Breugel 1992) but sometimes extending: beyond radio hot spots (van Breugel 1988). The alignment between the EELRs and radio sources becomes better for high redshift / more powerful objects (McCarthy 1989; Fig. 1).

\subsubsection{Luminosities}

The EELR and radio luminosities are suprisingly well correlated (Baum and Heckman 1989a,b; McCarthy 1989; McCarthy et al. 1991) This, together with 2.1.2., strongly suggests that the EELRs and radio lobes have a common source of energy (presumably the nucleus).

\subsubsection{Asymmetries}

EELR and radio lobe asymmetries are strongly correlated: the strongest line emission is always found on the side where the lobe is closest to the nucleus (McCarthy, ran Breugel and Kapahi 1991; Fig. 2). It suggests that the gaseous environments of radio galaxies are asymmetric, and that the radio lobes are slowed clown most on the side of the densest gas. As a corollary, this means that radio lobe distance asymmetries cannot be used to constrain lobe advance speeds and radio source ages. I am referring to the contribution by G. Pooley at this workshop for the discussion of observer asymmetries in radio lobe polarizations and spectral indices.

\subsubsection{Kinematics}

The kinematics of EELRs in nearby radio galaxies appears to be dominated by graritational forces, including rotation of gaseous disks acquired through merging with gas-rich companions or accretion from halos or cooling flows (e.g. Baum et al. 1991; Tadhunter 1991). However, radio jets/lobes can significantly affect the kinematics of emission-line gas at locations where they interact with ambient gas. Recent examples are Cygnus $\mathrm{A}$ and $3 \mathrm{C} 265$, with EELR velocities of as much as $1800 \mathrm{~km} \mathrm{~s}^{-1}$ (Tadhunter 1991). Other 

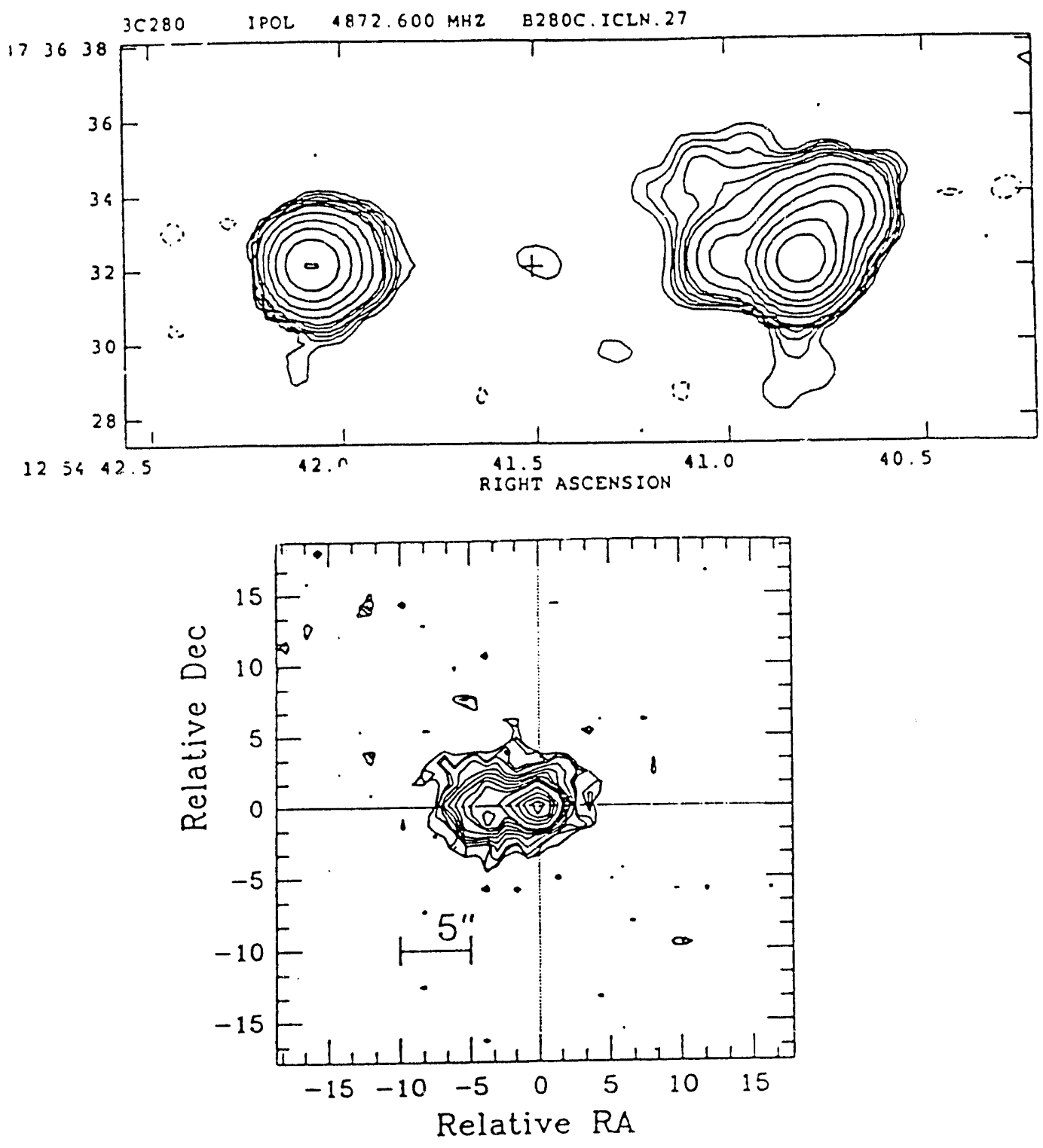

Fig. 2. An example of the correlation between EELR and radio lobe asymmetries (NcCarthy, van Breugel and Kapahi 1991): shown are a $6 \mathrm{~cm}, 1^{\prime \prime}$ resolution VLA map of 3 C $280, z=0.99$ (top), and a continuum subtracted narrowband [OII] 33727 image (bottom). Note that EELR is on the side with the lobe nearest to the nucleus (lob: distance ratio $1 / 1.2 T$ ).

examples are $3 \mathrm{C} 277.3,4 \mathrm{C} \geq 9.30$ and $3 \mathrm{C} 321$. and it is worth repeating (see van Breugel 1988) that the velocities of emission-line gas seem to be nearly always blue-shifted on the side of the brightest jets. This might mean that these jets are Doppler boosted and pointed towards us. The question remains. however: where are the redshifted EELRs near radio knots of unseen (counter-) jets. Which in such models should also exist and be pointed away from us? It is clearly of interest to continue studies of the kiriematics of EELRs associated with radio jets. The emission-line kinematics near hotspots may also be interpreted in terms of gas entrainment by lobes propagating through dense gas (e.g. 
van Brengel et al. 1985a [3C 277.3]; IVilson and Uilvestad 1987 [NGC 1068]; Pedlar et al. $10 S 9$ [Scyfert's]). A nice and detailed interpretation of the kinematics of emission-line gas entrained by the radio lobes of $3 \mathrm{C} 368$ has been presented by $\mathrm{K}$. Meisenheimer (this workshop).

\subsubsection{Ionization}

Quite generally, the emission-line ratios of EELRs, both in nearby and distant radio galaxies, can be adequately modelled with simple photoionization models in which the radio galaxy nuclei are also sources of jonicing (UV. X-ray) radiation directed or collimated in cone-like fashion along the same axes as their bulk outflow (jets, winds). I consider it beyond the scope of this review to give a complete discussion of the pros and cons of this "standard interpretation" except to mention that 1) the low ionization EELRs and their associated (?) low power radio sources in cooling flow clusters may. well require a different dominant source of ionization see (Heckman et al. 1986), 2) close scrutinity of emission line ratios reveals some inconsistencies which require either more sophisticated AGN photoionization models, or leave room for alternative explanations (i.e. shock ionization) in specific cases. Two such cases are discussed in detail by $\mathrm{K}$. Meisenheimer (3C 368 ) at this workshop.

\subsection{Extended Optical Continuum}

There is increasing evidence that the presence of very blue optical continuum emission associated with radio galaxies may be a relatively common phenomenon. The origin of this continuum may be young stars, scattered light or synchrotron emission, all of which are related to different aspects of the interaction of a radio source with its environment.

This is perhaps most dramatically illustrated by the discoveries that distant radio galaxies exhibit blue optical continuum which is nearly always aligned with the axes of their associated radio sources (McCarthy etal. 1987; Chambers et al. 1987; McCarthy et al. 1990, 1991). While it is likely that most of this optical emission is probably due to young stars (see for example Chambers and McCarthy 1990), optical polarization observations have shown that at least some of the UV excess may be due to scattered light from the nucleus (Scarrott et al. 1990: Januzzi and Elston 1991). Furthermore, as emphasized by Meisenheimer at this workshop, objects with very bright EELRs such as 3C 368 may significantly "contaminate" broadband images if strong emission-lines such as [OII] $\lambda 3727$ fall in the filter band pass. This is really only an issue for objects in the redshift range 0.6 - 1.1 (for example with the Lick/"Spinrad" $R_{s}$ filter covering 6000 - $7700 \AA$ ). and even in this redshift range emission-line free "intermediate broadband" images have confirmed the basic alignment effect (Rigler et al. 1992).

Also, in several nearby radio galaxies blue continum emission has been found. There are the wellknown cases of optical synchrotron knots associated with radio jets ( $M 87$, $3 \mathrm{C} \mathrm{66B}, 3 \mathrm{C} 277.3$, PIS 0521-36 etc.) and lobes (3C 33, Pictor A and others; see elsewhere in these proccedings and those of the first Ringberg workshop). Young stars and scattered continuum are not as commonly found, although some spectacular examples are known (Minkowski's Object, van Breugel et al. 1985b; PKS 2152-69, di Serego Alighieri et al. 1988). 

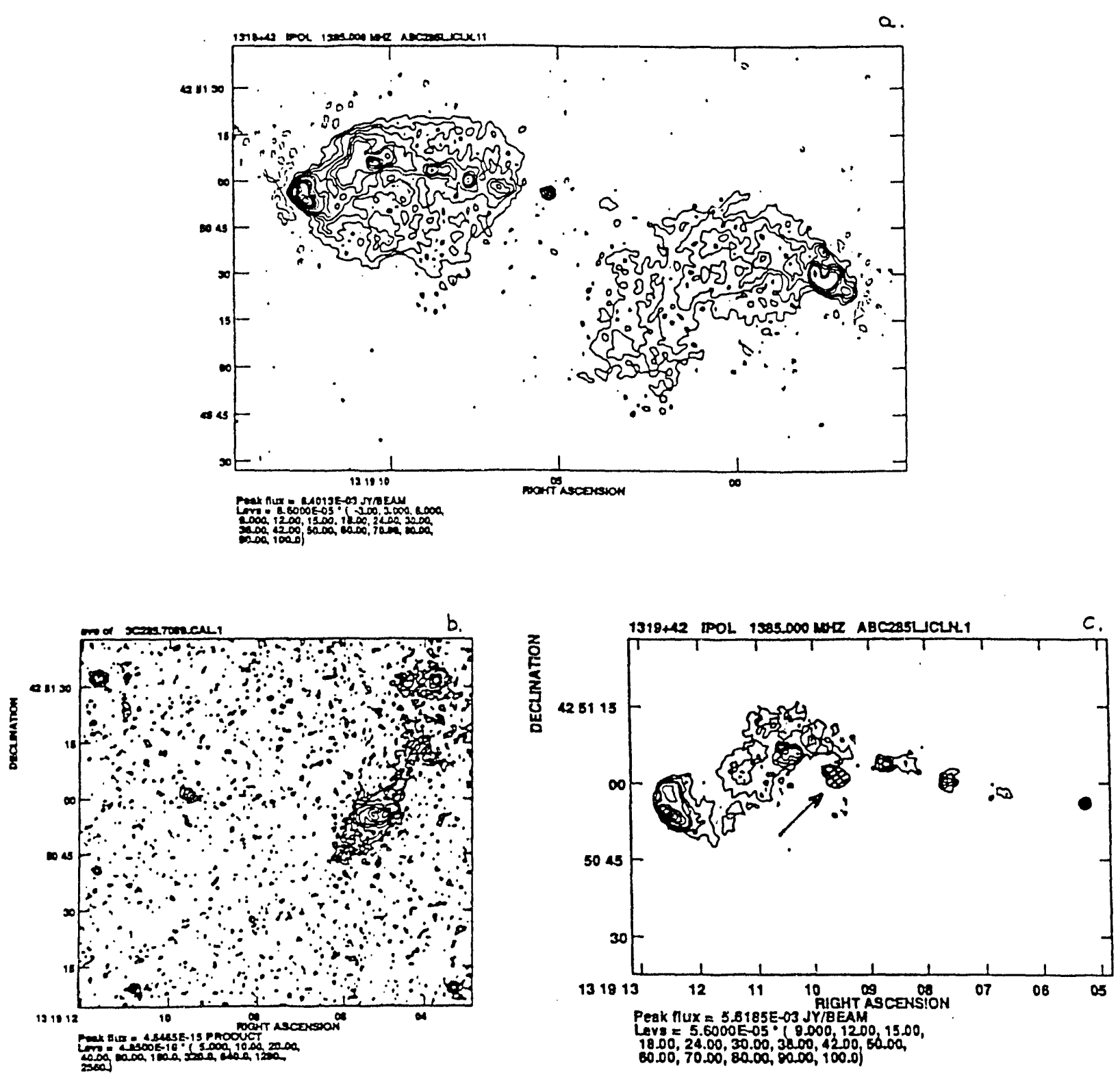

Fig. 3. Radio and optical images of $3 \mathrm{C} 28.5$ showing the location of a star forming region behind the eastern hot spot. Fig. 3 a shows a smoothed $2{ }^{\prime \prime} 1$ resolution $21 \mathrm{~cm}$ image of $3 \mathrm{C} 285$. Fig. $3 \mathrm{~b}$ shows a narrow-band image centered at the $\mathrm{H} \alpha$ emission-line (Baum et al. 1990; continuum not subtracted). Note the small. slightly extended EELR at $\sim 45^{\prime \prime}$ east of the parent galaxy. Lick spectra have shown that this EELR is an HII region at the redshift of $3 \mathrm{C} 28.5$. Its location relative to the radio hot spot is shown in Fig. 3c (see arrow).

Motivated by the rather general presence of UV continuum associated with distant radio galaxies, and by evidence that similar effects - on a more modest scale - also seem to occur in nearby, less powerful radio galaxies, A. Dey (UC Berkeley) and I are conducting a systematic optical $U$ - and $R$-band survey of a well defined sample of relatively nearby radio galaxies selected from the $3 C R$ and $P K S$ catalogs. All objects 
are constrained to have redshifts between $0.085-0.200$ so that no bright emission-lines fall in the $L^{-}-([\mathrm{OII}], 1372 T$ ) and $\mathrm{R}$ - ([OIII], $500 T$ ) passbands (when corrolved with the detector/telescope/atmosphere throughput).

Our first results have been rery encouraging and suggest that in as many as $30 \%$ of the objects extra-nuclear $[V$ continuum knots exist which may in some way be associated with their radio sources. One example is $3 \mathrm{C} 285$ (Fig. 3), which was already. known to have a rery blue compact object along its radio axis (Saslaw. Tyson and Crane 1978 ), at some $60 \mathrm{kpc}$ from the nucleus. Although noted by the authors it was not the main focus of their attention. which was aimed at the eastern "hot spot" of $3 \mathrm{C} 285$. Narrow-band imaging observations by Baum et al. 1990 of the 3C 285 field showed. by chance. that this compact. blue object also exhibits extended optical line emission, with the brightest line and continum emission facing the nucleus. Our spectroscopic observations at Lick have subsequently shown that it is an H-II region at the redshift of 3C 285. Thus, in many ways this object resembles Minkowski's Object. suggesting that the expanding radio lobes may have induced star formation in dense gas along the radio axis (possibly a cloud of cold gas or gas associated with a small, gasrich galaxy which happened to be in the path).

Of course, unlike in Minkowshi's Object and also NGC 7385 (Hardee, Owen and Eilek 1980), in 3C 285 there is no dire ${ }^{-+}$morphological association between the star forming region and a relatively bright radio component (jet or hot spot). This is perhaps not too surprising though since $3 \mathrm{C} 285$ is a nearly 100 times more powerful. FR II type radio source. Presumably its jet and hot spot propagation speeds are much larger than those of lower power. FR I type sources and any star forming regions triggered by its passage will correspondingly lag further behind. In fact, by studying the spectral energy distribution (SED) of this object one can in principle determine the age of its star burst using stellar population synthesis techniques (see for example Chambers and Charlot 1990). This in combination with the known (projected) distance behind the hot spot $(4.3 \mathrm{kpc})$ yields a new way of estimating the lobe advance speed, independicnt of the usual methods based on spectral ageing or ram pressure arguments (e.g. Leahy 1991).

We are currently still laching sufficient data for a detailed SED analysis, but it is probably safe to assume that the age of the starburst is somewhere between $10^{7}$ and $10^{8}$ years. Assuming, for example, an age of $j \times 10^{\top}$ years, we derive a hot spot adrance speed of $900 \mathrm{~km} / \mathrm{s}$. Allowing for projection effects, which may led to underestimate this velocity by a factor of 2 to 3 , and an uncertainty of a factor of 3 or so in the starburst age, which may go either way, I would conclude at this point that the hot spot speed is probably more than $1000 \mathrm{~km} / \mathrm{s}$, but probably less than $10,000 \mathrm{~km} / \mathrm{s}$. The upper estimate is consistent with the expansion speed derived for $3 \mathrm{C} 285$ from spectral ageing arguments ( $8400 \mathrm{~km} / \mathrm{s} ;$ Alexander and Leahy 1987 ). Studies of H-II regions behind radio hot spots in nearby FR II sources may thus provicle unique new constraints for radio galaxy models. 


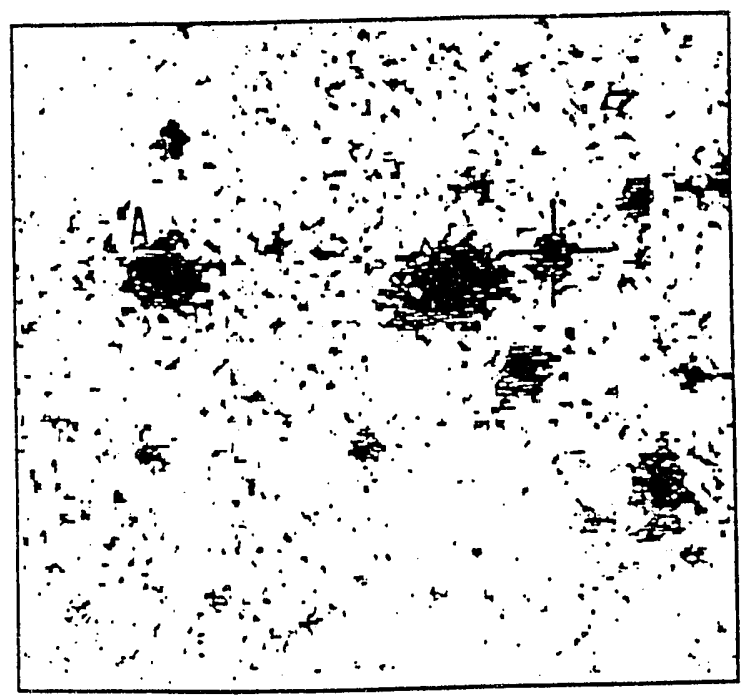

吕
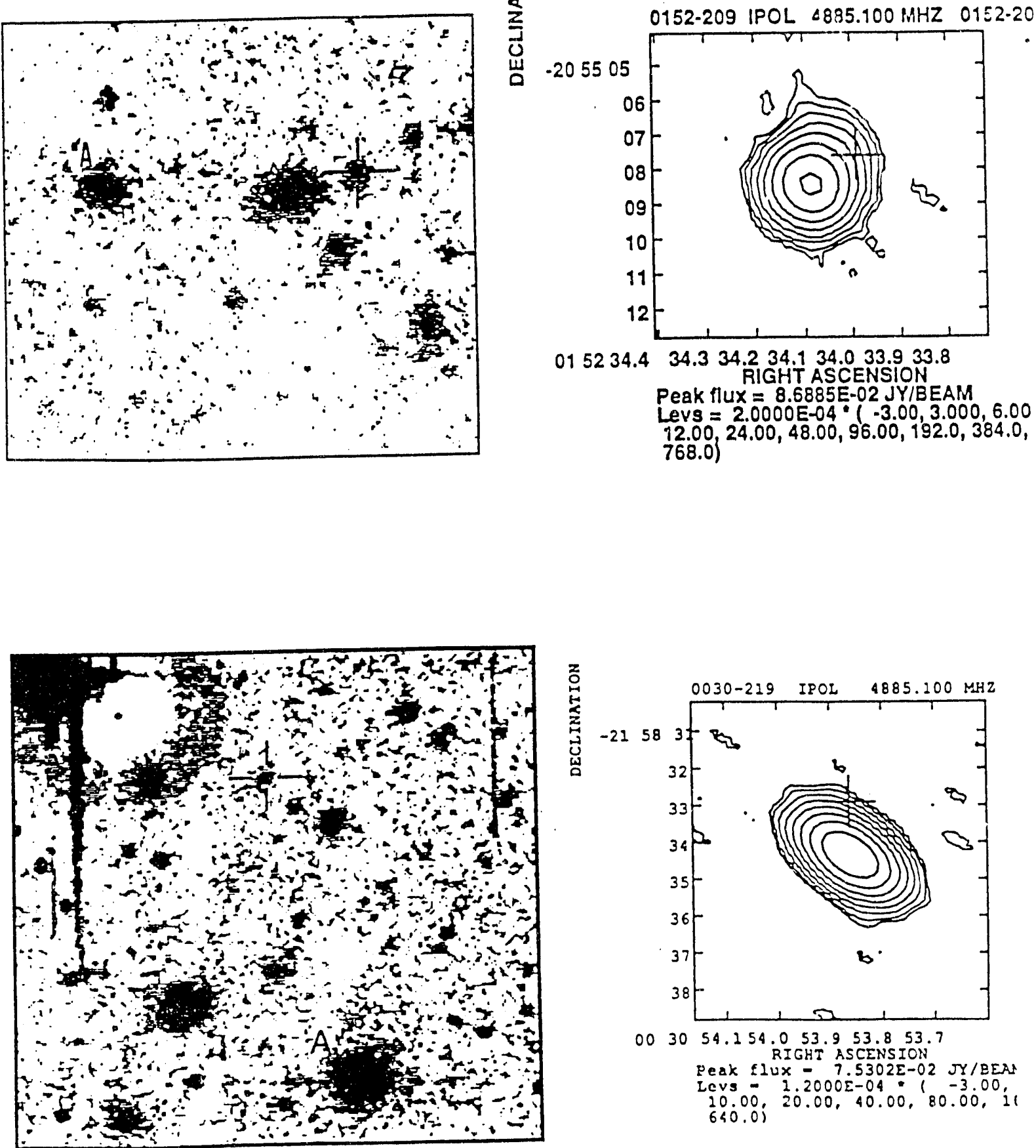

003054.154 .053 .953 .853 .7 peax ElUX $-7.5302 E-02$ TY/BEAS Levs $1000,2000 \Sigma-04.00,40.00,80.00$, i 640.00

Fig. 4. Two examples of high redshift radio galaxies with very compact parent galaxies (left hand panels; MRC 0030-219 and 0152-209 with $z=2.168$ and $z=1.590$ respectively') and equally compact steep spectrum radio sources (right hand panels; from McCarthy et al. 1990 , 1991). 


\section{Quasi-Stellar Distant Radio Galaxies}

While high redshift $(\because>1)$ radio galaxies generally have multi-modal, elongated morphologies more or less directed along their radio axes, it has also become clear from ungoing searches for distant radio galaxies that some of them can be rather compact or eren stellar in good seeing images. Two such examples are the Molonglo sources MRC 0152-209 (McCarthy et al. 1991) and MRC 0030-219 (McCarthy et al. 1990; Fig. 4). Interestingly, in both cases the associated radio sources are also very compact $\left(0^{\prime \prime} \cdot 6\right.$ and $<0$. 3 respectively). Their optical spectra show relatively narrow Ly- $\alpha$, indicating that these objects are galaxies, not quasars.

Another example' is MIRC 0349-211 ( $z=2.310 ;$ McCarthy et al. 1991). In this case, the galaxy is relatively compact but the radio source is fairly large ( $\tau^{\prime \prime} .4$ : $\left.94 \mathrm{kpc}\right)$, and has a relatively strong core. There is also evidence that the CIV 1549 line is fairly broad. Together these observations suggest that MRC 0349-211 might be an example of a high redshift "broad-line radio galaxy" (this class includes nearby sources such as 3C 390.3 and $3 \mathrm{C} 145$, and their parent " $N$-type" galaxies usually hare strong nuclear continua). I would conclude from these ongoing investigations that there is increasing evidence that distant radio galaxies may exhibit a wide variety of properties and it will be interesting to compare these with those of quasars at similar redshifts to investigate a possible connection, evolutionary or otherwise (e.g. Heckman et al. 1991; Neff and Hutchings 1990; Barthel 1989).

This work was performed under the auspices of the U.S. Department of Energy at the Institute of Geophysics and Planetary. Physics, Lawrence Livermore National Laboratory, under contract W-7405Eng-48.

\section{References}

Alexander, P., Leahy, J.P.: 19ST, Mon. Not. R. astr. Soc. 225, 1.

Barthel, P.D.: 1959, Astrophys.J. 336, 606.

Baum, S.A., Heckman, T.M., van Breugel, W.J.M.: 1990, Astrophys. J. Suppl. 74, 389.

Baum, S.A., Heckman, T.M.: 1989a, Astrophys. J. 336, 681.

- : 1989b, Astrophys. J. 336, 702.

Baum, S.A., Heckman, T.M., van Breugel, W.J.M.: 1992, Astrophys. J. 389, 208.

Chamhers, K.C., Miley, G.K., van Breugel, W.J.M.: 1987, Nature 329, 604.

Chambers, K.C., McCarthy, P.J.: 1990, Astrophys.J. (Lett.) 354, L9.

Chambers. K.C.. Charlot, S.: 1991, Astrophys. J. (Let.t.) 348, L1.

di Serego Alighieri, S. et al.: 1988, Nature 334, 591.

Hardee, P.E., Owen, F.N., Eilek, J.A.: 1980, Astrophys. J. 242, 502.

Heckman, T.M., Carty, T., Bothun, G.D.: 1985 Astrophys. J. 288, 122.

Heckman, T.M.: 1986, Astrophys.J. 311, 526.

- 1990 , in IAU Colloq. 12+ Paired and Interacting Galaxies, NASA Conf. Publ. 3098, eds. J.W. Sulentic, W.C. lieel and C.M. Telesco.

Heckman, T.M., Lehnert, M.D., van Breugel, IV.J.M., Miley, G.K.: 1991, Astrophys. J. 370, 78 .

Hernquist, L.: 1989, Nature 340,68T.

Hill, G.J., Lilly, S.J.: 1991. Astrophys. J. 367, 1.

Hutchings, J.: 19Si, Astrophys. J. 320, 522.

Januzzi, B.T., Elston, R.: 1991, Astrophys. J. (Let.t.) 366, I69.

Leahy, J.P.: 1991, in Beams Jets in Astrophysics, ed. P.A. Hughes, Cambridge: Cambridge University Press, p. 100. 
McCarthy, P.J.: 1989, Ph.D. Thesis, UC Berkeley.

McCarthy. P.J.. van Breugel. W..J.M.. Spinrad. H. Djorgorski. S.: 1997.

Astrophys. J. (Lett.) $\mathbf{3 2 1 . ~ L 2 9 . ~}$

McCarthy, P.J., Kapahi. V.K.. van Breugel, W.J.M., Subrahmanya. C.R.: 1990. Astron.J. 100.1014.

IicCarthy, P.J.. van Breugel. W.J.M., Kapahi, V.K., Subrahmanya, C.R.: 1991, Astron. J. 102, 522.

McCarthy, P.J.. van Breugel, W.J.M. Kapahi, V.K.: 1991, Astrophys.J. 371, 478.

MicCarthy, P.J., Spinrad, II., van Breugel, W.J.M.: 1993, Astrophys. J. Suppl., in press.

Neff, S.G., Hutchings. J.B.: 1990, Astron. I. 100, 1441.

Corman. C.A., Scoville, X.: 19SS. Astrophys. J. 332. 124.

Owe', F.N., Laing. R.: 1989, Mon. Not. $R$ astr. Soc. 238, 357.

Pedlar, A.: 1989 Mon. Not. R. astr. Soc. 238, 863.

Rigler. M.A.: 1992. Astrophys. J. 385. 61.

Saslaw. W.C., Tyson, J.A.. Crane, P.: 1978, Astrophys. J. 222,. 435.

Scarott, S.M., Rolph, C.D.. Tadhunter, C.N.: 1990, Mon. Not. R. astr. Soc. 243. 5P.

Shlosman, I., Begelman, M.C.. Frank, J.: 1990. Nature 345, 6 i9.

Smith, E.P., Heckman, T.M.: 1989, Astrophys.J. 341, 658.

- : 1990, Astrophys. J. 348, 38.

Smith, E.P., Heckman, T.M., Illingworth, G.D.: 1990, Astrophys. J. 356, 399.

iadhuntr:, C.N.: 1991, Mon. Not.R. astr. Soc. 251, 46P.

van Breugel, W.J.M.: 1985, Astrophys.J. 290, 496.

- : 1985, Astriphys. J. 293. 83.

- 1988, in Hot Spots in Extragalactic Radio Sources, Lecture Notes in Physics 327, eds. K. Meisenheimer and H.-J. Röser, Heidelberg: Springer Verlag: p. 121.

Toomre, A., Toomre, J.: 1972. Astrophys. J. 178, 623.

Wilson. A.S., Vilvestad, J.S.: 1987, Astrophys. J. 319, 105.

lates, M.G., Miller, L., Peacock. J.A.: 1989, Mon. Not. R. astr. Soc. 240, 129. 

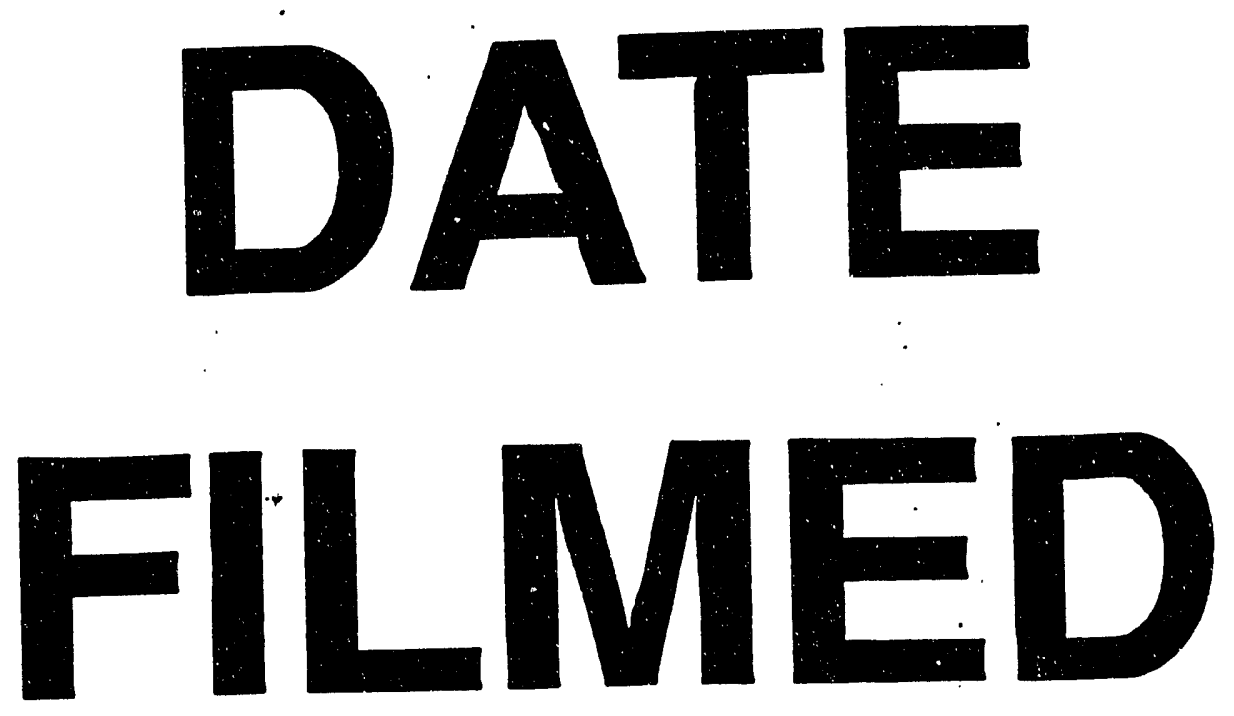

$12 / 6 / 93$
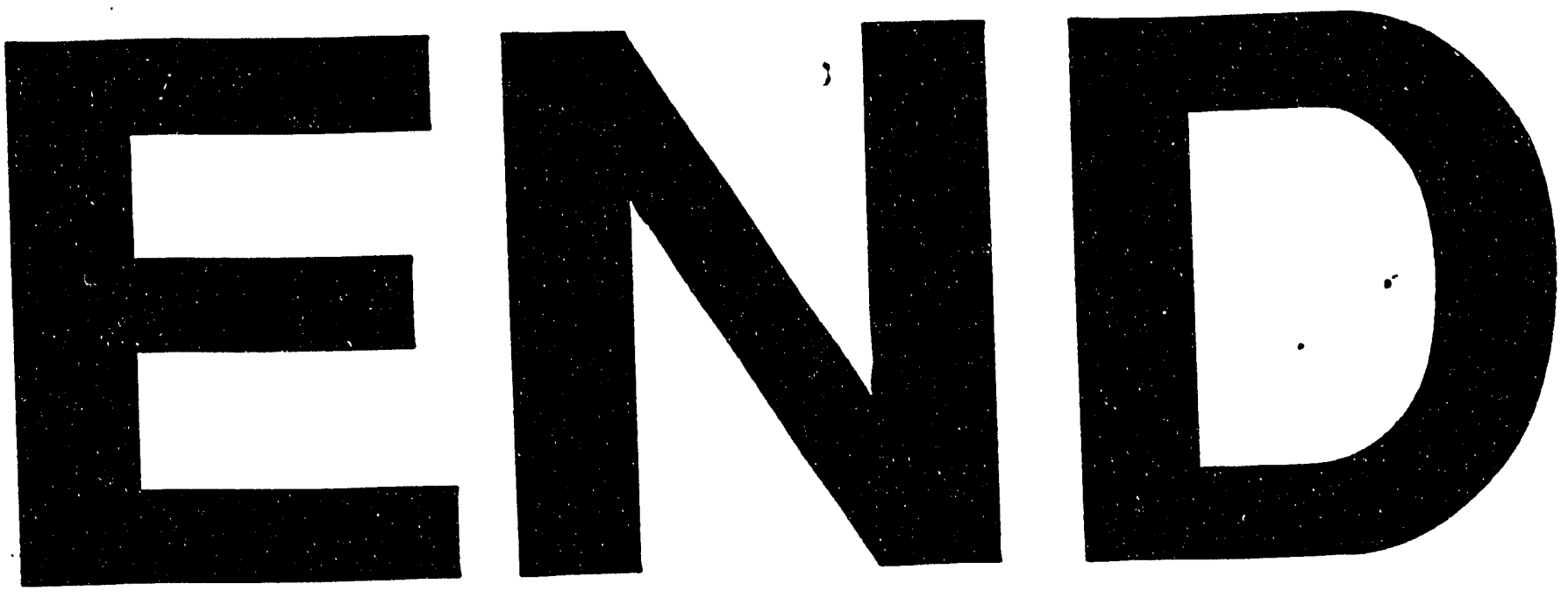
